\title{
Group Invariant Solutions Derived from Equivalent Lagrangian in a Class of Kuramoto Sivashinsky (KS) Equations
}

\author{
J E Okeke, O C Collins
}

\begin{abstract}
The method of equivalent Lagrangians yields transformation maps which can be used to find solutions and conserved quantities of a class of Kuramoto Sivashinsky equations. Furthermore, we utilize the Lie algebra admitted by the equation to obtain an optimal system of one dimensional subalgebras for the equation. All equivalent invariant solutions corresponding to these subalgebras are presented.
\end{abstract}

Index Terms - Equivalent Lagrangian, Symmetries, and optimal system.

\section{INTRODUCTION}

It has been shown that the notion of equivalent Lagrangians are used to construct Lagrangians for differential equations with a known Lie algebra of point symmetries (Kara, 2004; Okeke \& Laisin, 2017), where the differential equations are derivable from a variational principle (i.e., differential equations that have Lagrangians). The significant of this method is to find the solutions of a given differential equation that has an isomorphic algebra of both the Lie point and Noether point symmetries. This means that the algebra of Noether point symmetries admitted by the Lagrangian and its equivalent must be of the same dimension. The same applies to the algebra of lie point symmetries of the given differential equation and the resultant equation from the equivalent Lagrangian. The method involves the construction of a regular point transformation which maps the Lagrangian of a simpler differential equation with known solutions to the Lagrangian of the equation we seek to solve (Wilson \& Kara, 2012). Once this transformation is found, one can map the solutions of the simpler equation to solutions of the given equation we want to solve, provided the earlier mentioned properties are satisfied by the equation.

The application of the concept of equivalent Lagrangians to construct Lagrangians for differential equations with a known Lie algebra of point symmetries has recently been a subject of extensive study. For example, Kara and Mahomed (Kara \& Mahomed, 1992) applied the method to two cases of the equation of the form

$\ddot{q}+p(t) \dot{q}+r(t) q=\mu \dot{q}^{2} q^{-1}+f(t) q^{n}$.

J E Okeke, Department of Mathematics, Chukwuemeka Odumegwu Ojukwu University, Anambra State, Nigeria,

O C Collins, Department of Mathematics, University of Nigeria, Nsukka, Enugu State, Nigeria
Kara (Kara, 2004, ) used the approach to derive equivalent Lagrangians for a unit second order wave equation and a system of second order ordinary diff erential equations. In this paper, we extend the application to a fourth order partial diff erential equation of the form

$U_{\mathrm{tt}}+\alpha U_{x x x x}-\gamma\left(U_{x}^{\mathrm{n}}\right)_{x}=0$

where $\alpha, \gamma$ are constants and $n>0$. This equation (2) is a modified nonlinear wave equation introduced by Yang and Chen (Yang et al., 2000). It is associated with many equations. For example, in [Peire et al., 1995) a nonlinear wave equation.

$U_{t t}+\alpha U_{x x x x}-\gamma\left(U_{x}^{2}\right)_{x}=0$,

where $\mathrm{u}(\mathrm{x}, \mathrm{t})$ is the longitudinal displacement, $\alpha>$ $0, \gamma \neq 0$ are real numbers was presented. It is used to study some problems about vertical vibration of one dimensional elasticity pole and two dimensional anti-plane shear in the weak nonlinear analysis of micro-structure model in the elasticity and plasticity. Furthermore, the instability of its special solution and ordinary stain solution were studied (Peire \& An, 1995). Chen and Yang (Chen, \& Yang, 2000) and Zhang and Chen (Zhang \& Chen, 2003) considered the generalized equation of equation (3) and proved the existence and uniqueness of the global generalized solution and the global classical solution of several initial boundary value problems by the contraction mapping principle. The sufficient conditions of the nonexistence of the solution were also given. Yan (Yan, 2000) studied the equation (2) with the viscous damping term, by using the direct reduction method and obtained four new explicit solutions in the case of $n=2$. The work of Yan (Yan, 2000) was extended by Wu and Fan (Wu \& Fan, 2007) via the same method and presented the solutions for the equation for $n \geq 3$. However, none of these studies categorizes analytic, exact or invariant solutions that are related to the symmetries of the equation (2) In this paper, an attempt at an analysis of these aspects of the equation is carried out to investigate more new solutions of the equation. The aim of this paper therefore is first to construct equivalent Lagrangians of equation (2) from which the solutions of the equation can be found if the solutions of the equation associated with the equivalent Lagrangians are known or vice versa, and obtain exact invariant solutions of the equation.

The outline of the paper is as follows. In the next section, we present some basic operators, definitions and concept of equivalent Lagrangians. In Section III, the equivalent 
Lagrangians of the nonlinear wave equation (2) are constructed through point transformations of the symmetries. In Section IV, the optimal system of one dimensional subalgebras of the Lie symmetries of (2) is derived. Moreover, using the optimal system of subalgebras, symmetry reductions and equivalent group invariant solutions are obtained in section V. A brief discussion and conclusion is given in the last Section.

\section{PRELIMINARIES}

\section{Definition 2.1.}

A $k^{\text {th }}$ - order $(\mathrm{k} \geq 1)$ System $E^{\sigma}$ of s partial diff erential equations of $n$ independent variables $x^{i}{ }_{x} i=1_{x} 2_{\ldots \ldots} n$ and $m$-dependent variables $u^{\alpha}: \alpha=1,2_{y \ldots} m$ is defined by;

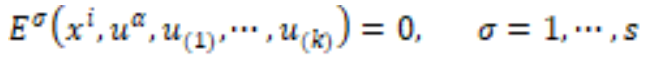

where $u_{(1), \ldots,} u_{(k)}$ denote the collection of all first, second, ..., kth-order partial derivatives.

\section{Definition 2.2.}

The Euler-Lagrangian operator is defined by

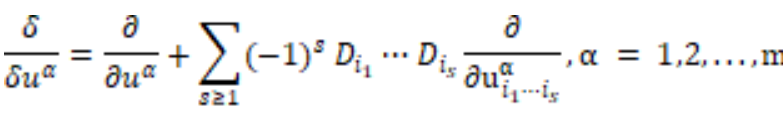

where

$D_{i}=\frac{\partial}{\partial x^{i}}+u_{i}^{a} \frac{\partial}{\partial u^{x}}+u_{i j}^{a} \frac{\partial}{\partial u_{j}^{\alpha}}+\cdots x_{x} \quad i=1,2 \ldots \ldots x n$

is the total derivative operator with respect to $x^{i}$

\section{Definition 2.3.}

The Euler-Lagrangian equations, associated with (4) are the equations

$\frac{\delta L}{\delta u^{\alpha}}=0, \quad \alpha=1,2 \ldots \ldots \mathrm{m}$

where $\mathrm{L}$ is referred to as a Lagrangian of (4).

\section{Definition 2.4.}

A Lie Backlund operator $\mathrm{X}$ is defined by

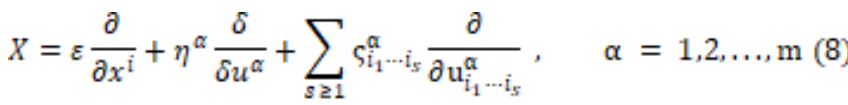

where $\varsigma_{i_{1}-\cdots i_{\mu}}^{\mathbb{a}}$ are given as

$\varsigma_{i}^{\alpha}=D_{i}\left(\eta^{\alpha}\right)-\varsigma_{i_{1} \cdots i_{x}}^{\alpha} D_{i} \varepsilon^{j}, \quad \varsigma_{i_{1} \cdots i_{x}}^{\alpha}=D_{i_{x}}\left(\varsigma_{i_{1} \cdots i_{x}}^{\alpha}\right)-u_{j_{i_{1}} \cdots i_{x-1}}^{\alpha} D_{i_{u}}\left(\varepsilon^{j}\right), s \geq 1$

Definition 2.5. A Lie Backlund operator $X$ of the form (8) is called a Noether symmetry generator associated with a Lagrangian $L$ of (7) if there exists a vector $B=\left(B^{1}, B^{2}, \ldots, B^{n}\right)$ such that
$X L+L D_{i}\left(\varepsilon^{i}\right)=D_{i}\left(B^{i}\right)$

where $\mathrm{X}$ is prolonged to the degree of L (Olver, (1993). If the vector B is identically zero, then $\mathrm{X}$ is astrict Noether symmetry (Ibragimov et al., 1998). For each Noether symmetry generator $\mathrm{X}$ associated with a given Lagrangian $\mathrm{L}$ corresponding to the Euler-Lagrange diff erential equations, a conserved quantity is obtained (Kara et al., 2009; Okeke, Narain \& Govinder, 2018) using the equation

$T^{i}=B^{i}-N^{i} L_{s} \quad i=1,2 \ldots \ldots s n$

Definition 2.6. Two Lagrangians, $\bar{L}=\bar{L}\left(x, u_{w} u_{[\mathbb{1})}, \cdots, u_{(y)}\right)$ and $L=L\left(X, U_{v} U_{\left.(\mathbb{1}) s^{\cdots \times}, U_{(x)}\right)}\right)$, are said to be equivalent if

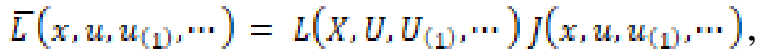

where $X=X(x, w), U=U(x, u)$ and $l$ is the determinant of the Jacobian matrix (Kara et al., 2009).

For ordinary diff erential equations in which $u=u(x)$, the definition of equivalence up to gauge function, $f=(x, u)$ is given as

Definition 2.7. Two Lagrangians, $\mathrm{L}$ and $\bar{L}$, are said to be equivalent up to gauge function,

$f=(x, u)$ if

$\bar{L}\left(x, u, u^{I}\right)=L\left(X, U, U^{I}\right) \frac{d X}{d x}+f_{x}+u^{I} f_{u}$

where

$X=X(x, u)$ and $U=U(x, u)$ (Kara \& Mahomed, 1992).

\section{EQUIVALENT LAGRANGIAN FOR THE CLASS OF KS EQUATIONS}

Firstly, we present the Lie and Noether point symmetries of (2) which shall be used in this section to form the equivalent Lagrangian of the equation and in subsequent sections for further analysis. The Lie point symmetry generators of (2) is a five-dimensional Lie algebra spanned with the following basis $X_{1}=\frac{\partial}{\partial x^{x}}, X_{2}=\frac{\partial}{\partial t^{x}}, X_{3}=\frac{\partial}{\partial u^{x}}, X_{4}=t \frac{\partial}{\partial u}, X_{5}=\frac{x}{2} \frac{\partial}{\partial x}+$
$t \frac{\partial}{\partial t}+\frac{\mu u}{2} \frac{\partial}{\partial u^{x}} \quad$ (14)

where $\mu=\frac{n-3}{n-1}$. Obviously, when $n=1$, the dilations in space and time are lost. This seems outstanding and distinguishes the symmetry structure of (2) for $n=1$ from any other values of $n$. The Noether point symmetries of the Lagrangian

$L=\frac{1}{2} u_{t}^{2}-12 \alpha u_{x x}^{2}-\frac{1}{n+1} \gamma u_{x}^{n+1}$

of equation (2), are the generators $X_{1}$ to $X_{4}$ above, all having zero gauge functions except $X_{4}$ which has a gauge 
function $\left(B_{1}, B_{2}\right)=(u, 0)$.We want to construct a Lagrangian $\bar{L}=\bar{L}(r, s, v), v=v(r, s)$ equivalent to $(15)$ using the transformation

$x=x(r, S, v), t=t\left(r, r_{v} v\right), u=u(r, S, v)$. Since any one parameter group $\mathrm{G}$ of a transformation can be reduced under a suitable change of variables to the translation group with the operator $G=\frac{\partial}{\partial t}$ (Ibragimov, 1994), suitably equivalent quantities can be constructed using symmetry structures. Therefore, a point transformation that leaves the Lagrangians of (2) invariant under change of variables can be obtained through point transformations of its symmetry structures. Hence, by mapping a Noether symmetry generator

$$
X_{2}=\frac{\partial}{\partial t}
$$

to the dilation operator in $\left(r_{v} s, v\right)$ variables

$$
X_{2}=\frac{1}{2} r \frac{\partial}{\partial t}+s \frac{\partial}{\partial s}+\frac{1}{2} \mu v \frac{\partial}{\partial v}
$$

from the Lie point symmetries (14), we obtain the point transformation $x=f\left(\frac{s}{r^{2}}, v r^{-\mu}\right)$.

$t=\log r+g\left(\frac{s}{r^{2}}, v r^{-\mu}\right), \quad u=h\left(\frac{s}{r^{2}}, v r^{-\mu}\right)$ As an

example, we let

$x=\frac{s}{r^{2}}, \quad t=\log r_{v} \quad u=v r^{-\mu}$

Thus, it follows that

$u_{x}=r^{2-\mu_{v_{s}}} u_{t}=2 s r^{-\mu_{v_{g}}}+r^{1-\mu_{v_{r}}}-$

$\mu r^{-} \mu_{v_{s}} u_{x x}=r^{4-\mu_{1}} v_{s s}$

and the Jacobian of the transformation $J=-\frac{1}{x^{3}}$. By

Definition 2.6, a Lagrangian equivalent to (15) is of the form $\bar{L}=\frac{1}{r^{3}}\left[\frac{1}{2}\left(2 s r^{-} \mu_{v_{s}}+r^{1-\mu_{v_{r}}}-\mu r-\mu_{v}\right)^{2}-\right.$

$\left.\frac{1}{2} \alpha\left(r^{8-2 \mu_{v_{s g}}^{2}}\right)-\frac{1}{n+1} \gamma\left(r^{2-\mu_{v_{s}}}\right)^{n+1}\right]$

The Euler Lagrangian equation associated with equation (19) is

$$
\mu^{2} v v_{s}-4 s(\mu-1) v_{g}^{2}-n \gamma r^{n+5} v_{g} v_{s g}+v_{s}\left(4 s^{2} v_{s g}+\right.
$$$$
\left.r\left(r^{7} \alpha v_{s s s}+(1-2 \mu) \nu_{r}+4 s v_{r s}+r v_{r r}\right)\right)=0, \text { (20) }
$$

To verify that this Lagrangian (19) is indeed equivalent to (15) under the point transformation (18), we calculate its Noether point symmetries in the new variables $(r, s, v)$ given as

$$
\begin{aligned}
& X_{1}=r^{2} \frac{\partial}{\partial s}, B^{1}=0, B^{2}=0 \\
& X_{2}=r \frac{\partial}{\partial r}+2 s \frac{\partial}{\partial s}+\mu v \frac{\partial}{\partial v}, B^{1}=0, B^{2}=0 \\
& X_{2}=r^{\mu} \frac{\partial}{\partial v}, B^{1}=0, B^{2}=0 \\
& X_{4}=r^{\mu} \log r \frac{\partial}{\partial v}, B^{1}=r^{-\mu_{v}}, B^{2}=0
\end{aligned}
$$

Clearly, this Noether algebra (21) is isomorphic to the Noether algebra of the Lagrangian L of (15) Hence, L and $\bar{L}$ are equivalent under the point transformation (18). Thus, using the equivalent Lagrangian approach, we derive new wave equation (20) from (2) which has some physical interpretations in physics but appears complex. However, its solutions can be obtained using the transformation (18) once the solutions of the wave equation (2) are known. Many Lagrangians equivalent to (15) can also be constructed through mappings of other symmetry generators using similar approach.

\section{OPTIMAL SYSTEM OF SUBALGEBRAS}

The Lie group theory method plays an important role in finding exact solutions and symmetry reductions of differential equations. As any linear combination of symmetry generators is also a symmetry generator, there exist infinitely many different symmetry subgroups for a differential equation. Therefore, a method determining which subgroups would give basically different types of solutions is necessary and significant for a complete understanding of the invariant solutions.

In this section we discuss in brief the optimal system method proposed by Olver (Olver, 1993) to obtain one dimensional subalgebras of the symmetry group admitted by equation (2) which shall be used in the next section for reduction and construction of invariant solutions for the equation. In constructing one dimensional optimal system of symmetry group

$$
\begin{array}{r}
<X_{1}, X_{2}, X_{a}, X_{4}, X_{5}>\text {, we consider the general operator } \\
\qquad X=a_{1} X_{1}+X_{2}+X_{a}+X_{4}+X_{5 x}
\end{array}
$$

where $a_{i}{ }^{2} s$ are arbitrary constants. We apply adjoint maps to $\mathrm{X}$ in such a way as to simplify and/or eliminate as many of the coefficients $a_{i}{ }^{2} s$ as possible to obtain a new simpler operator. To compute the adjoint representation, we use the Lie series:

$$
A d\left(e^{\in X_{i}}\right) X_{j}=X_{j}-\in\left[X_{i j}, X_{j}\right]+\frac{1}{2 !} \epsilon^{2}\left[X_{i},\left[X_{i}, X_{j}\right]\right]-x_{x}
$$

where $i_{v} j$ take the values from 1 to 5 . Taking into account Table 1 for commutators, we obtain the adjoint Table 2. In what follows, after some calculations, it turns out that the optimal system of one dimensional subalgebras of (2) is $X_{1}, X_{2} \pm X_{1}, X_{2} \pm X_{1}, a X_{1}+b X_{2}+X_{4}, X_{5}$ where $a, b$ are arbitrary constants. Now using the discrete symmetry $x \rightarrow-x$ of (2), we can delete the operators $X_{2}-X_{1}$ and $X_{a}-X_{1}$ from the set. Hence, finally, the optimal system of one dimensional subalgebras is

$$
X_{1}, X_{a}+X_{1}, X_{2}+X_{1}, a X_{1}+b X_{2}+X_{4}, X_{5}
$$

Table 1: The commutation relations satisfied by generators (14)

\begin{tabular}{llllcl}
\hline []] & $X_{1}$ & $X_{2}$ & $X_{a}$ & $X_{4}$ & $X_{5}$ \\
\hline$X_{1}$ & 0 & 0 & 0 & 0 & $\frac{1}{2} X_{1}$ \\
$X_{2}$ & 0 & 0 & 0 & $X_{a}$ & $X_{2}$ \\
$X_{a}$ & 0 & 0 & 0 & 0 & $\frac{1}{2} \mu X_{a}$ \\
$X_{4}$ & 0 & $-X_{a}$ & 0 & 0 & $\frac{1}{2}(\mu-2) X_{4}$ \\
$X_{5}$ & $\frac{1}{2} X_{1}$ & $-X_{2}$ & $-\frac{1}{2} \mu X_{a}$ & $-\frac{1}{2}(\mu-2) X_{4}$ & 0
\end{tabular}


Table 2: Table of adjoint representation for (14)

\begin{tabular}{llll} 
Ad & $X_{1}$ & $X_{2}$ & $X_{a}$ \\
\hline$X_{1}$ & $X_{1}$ & $X_{1}$ & $X_{1}$ \\
$X_{2}$ & $X_{1}$ & $X_{2}$ & $X_{a}$ \\
$X_{a}$ & $X_{1}$ & $X_{2}$ & $X_{a}$ \\
$X_{4}$ & $X_{1}$ & $X_{2}+\in X_{a}$ & $X_{a}$ \\
$X_{5}$ & $e^{\frac{1}{2}} X_{1}$ & $e^{€} X_{2}$ & $e^{\frac{1}{2} \mu \Subset} X_{a}$
\end{tabular}

Table 3: Subalgebras, group invariant solutions and equivalent group invariant solutions

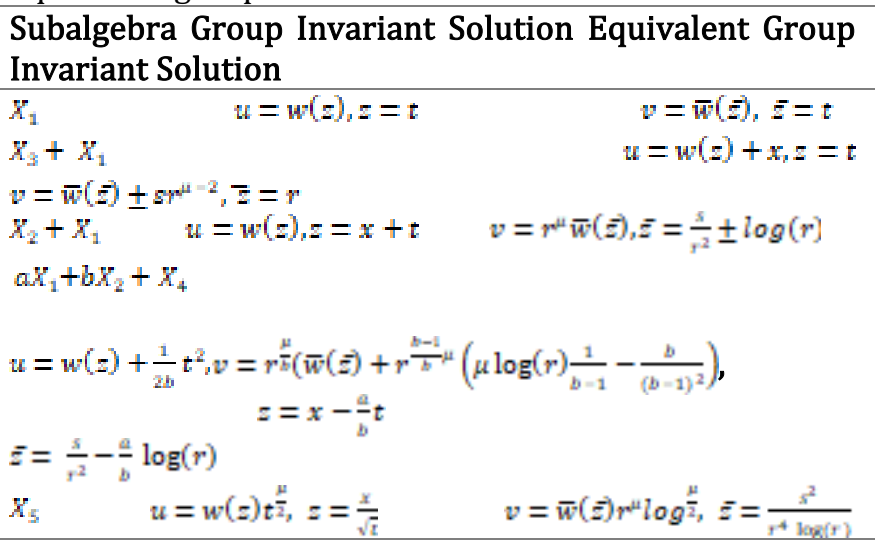

\section{SYMMETRY REDUCTION AND EQUIVALENT INVARIANT SOLUTION}

One of the main objectives for calculating symmetries of differential equations is to use them to reduce the differential equations which could be solved to obtain exact solutions. The optimal system of one dimensional subalgebras (24) obtained in Section 4 is used to reduce (2) to ordinary differential equations and find their exact solutions where possible. The group invariant solutions corresponding to the subalgebras are determined and presented in Table 3 . The equivalent group invariant solutions obtained using the transformation (18) are also presented.

V(a) Invariance under $X_{1}$ :

The substitution of the group invariant solution of $\boldsymbol{X}_{\mathbf{1}}$ into (2) gives rise to an ordinary differential equation whose general solution is the linear function in $\mathrm{t}$,

$$
u(t, x)=c_{1} t+c_{2} \text {. }
$$

where $\boldsymbol{c}_{1}, \boldsymbol{c}_{\mathbf{2}}$ are constants.

V(b) Invariance under $X_{3}+X_{1}$ :

Similarly, in this case the group invariant solution corresponding to the symmetry generator

$\boldsymbol{X}_{3}+\boldsymbol{X}_{1}$ leads to the linear solution in $\mathrm{t}$ and $\mathrm{x}$ variable,

$$
u(t, x)=c_{1} t+x+c_{2 x}
$$

where $\boldsymbol{c}_{1}, \boldsymbol{c}_{2}$ are constants.

V(c) Invariance under $X_{2}+X_{1}$ :

The group invariant solution of the subalgebra $X_{2}+X_{1}$ reduces (2) to
Solving (27), we obtain the solution of (2) in terms of Hypergêemetric function given as $u(t, x)$

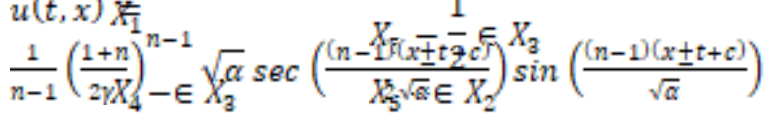

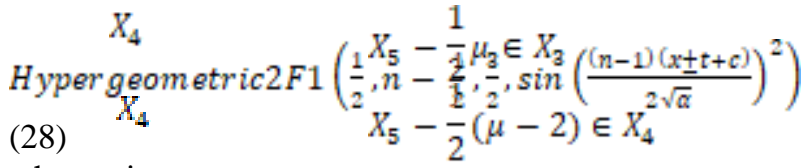

where $\mathrm{c}$ is a constant.

V(d) Infvarianceunder $a X_{1}+b X_{2}+X_{4}$ :

The group invariant solution arising from the subalgebra $a X_{1}+b X_{2}+X_{4}$ results to the following nonlinear ordinary differential equation

$$
\frac{1}{b}+\left(\frac{1}{b}\right)^{2} w^{H I}+\alpha w^{m E}-n \gamma w^{m-1} w^{H}=0 .
$$

Integrating (29) once and letting $w^{\prime}=\Psi$ yields a second order equation

$$
\frac{z}{b}+\left(\frac{1}{b}\right)^{z} \Psi+\alpha \Psi^{n}-n \gamma \Psi^{n}+c=0 .
$$

known as the first Painlevé transcendent. Its solutions $\psi=\sigma(z)$ are meromorphic in the entire complex plane, but are essentially new functions that cannot be expressed in any standard form (Olver, 1993). The corresponding solutions of the nonlinear equation (2) take the form

$\mathrm{u}(\mathrm{t}, \mathrm{x})=\mathrm{H}\left(\mathrm{x}-\frac{\mathrm{a}}{\mathrm{b}} \mathrm{t}\right)+\frac{1}{2 b} \mathrm{t}^{2}$.

where $\mathrm{H}=\int \sigma(z) d z$.

V(e) Invariance under $X_{5}$ :

The substitution of the group invariant solution of the symmetry $\boldsymbol{X}_{\mathbf{5}}$ into (2) yields the following nonlinear ordinary differential equation

$$
\begin{aligned}
& \frac{1}{4} \mu(\mu-2) \mathrm{w}-\frac{1}{2}\left(\mu+\frac{9}{2}\right) \mathrm{z}^{n}+\frac{1}{4} z^{2} \mathrm{w}^{n}+\alpha \mathrm{w}^{m !}- \\
& \mathrm{n} \gamma \mathrm{w}^{m-1} \mathrm{w}^{n}=0 .
\end{aligned}
$$

\section{CONCLUSION}

One of the main objectives for calculating symmetries of differential equations is to use them to reduce the differential equations which could be solved to obtain exact solutions. The optimal system of one dimensional subalgebras (24) obtained in Section 4 is used to reduce (2) to ordinary differential equations and find their exact solutions where possible. The group invariant solutions corresponding to the subalgebras are determined and presented in Table 3 . The equivalent group invariant solutions obtained using the transformation (18) are also presented.

\section{REFERENCES}

[1] Chen, G.W., \& Yang, Z.J. (2000). Existence and Nonexistence of Global Solutions for a Class of Nonlinear Wave Equations. Mathematical Methods of Appllied Science, 23, 615-631.

[2] Ibragimov, N. H. (1994). CRC Handbook of Lie Group Analysis of Differential Equations,vol.1, Symmetries and Exact Solutions and Conservation Laws, Boca Raton, Fla, USA.

[3] Ibragimov, N.H., Kara, A.H., \& Mahomed, F. M. (1998). Lie-Backlund and Noether Symmetries with Applications, Nonlinear Dynamics, 15 $115-136$. 
[4] Kara, A.H. (2004). Equivalent Lagrangians and the Inverse Variational Problem with Applications, Quaestiones Mathematicae, 27, 207-216.

[5] Kara, A. H. Bokhari, A.H., Karim, M., \& Zaman, F. D. (2009). Invariance Analysis and Variational Conservation Laws for the Wave Equation on Some Manifolds. International Journal of Theoritical Physics, 48, 1919-1928

[6] Kara, A.H., \& Mahomed, F.M. (1992). Equivalent lagrangians and the Solution of some Classes of Nonlinear Equations $\ddot{q}+p(t) \dot{q}+r(t) q=\mu \dot{q}^{2} q^{-1}+f(t) q^{n}$ International Journal of Nonlinear Mechanics, 27, 919-927.

[7] J. E. Okeke, R. Narain and K. S. Govinder, (2018). New exact solutions and conservation laws of a class of Kuramoto Sivashinsky (KS) equations; Quaestiones mathematicae 1-20, DOI: 10.2989/16073606.2018.1439542

[8] J. E. Okeke and M. Laisin, (2017) Equivalent Lagrangian and Lie Symmetry Analysis of a Class of Kuramoto Sivashinky (KS) Equations; Transactions of the Nigerian Association of Mathematical Physics Volume 5,(September and November, 2017), pp61 - 64 Volume 5, pages 61-64

[9] Olver, P. J. (1993). Applications of Lie Groups to Differential Equations, Springer, New York, NY, USA.

[10] Peire, A., An, L.J. (1995). A Weakly Nonlinear Analysis of Elasto-Plastic-Microstructure Models. SIAM Journal of Appllied Mathematics, 55 136-155.

[11] Wilson N., \& Kara, A.H. (2012).Equivalent lagrangians: Generalization,Transformation Maps, and Applications, Journal of Appllied Mathematics, doi:10.1155/2012/860482.

[12] Yan, Z.Y. (2000). Similarity Reductions for a Nonlinear Wave Equation with Damping Term. Acta Physica Sinica, 49, 2113-2117 in Chinese.

[13] Wu, H., \& Fan, T. (2007). New Explicit Solutions of the Nonlinear Wave Equations with Damping. Journal of Applied Mathematics1, 91, 457-465.

[14] Yang, Z.J., Chen, G.W. (2000). Initial Value Problem for a NonlinearWave Equation with Damping Term, Acta Mathematicae Applicae Sinica, 23 (45), in Chinese.

[15] Zhang, H.W., \& Chen, G.W. (2003). Potential Well Method for a Class of Nonlinear Wave Equations of Fourth-Order. Acta Mathematicae Science, 23A, 758-768. 